\title{
PRÉCISION D'UN SCHÉMA ADAPTATIF SEMI-LAGRANGIEN POUR L'ÉQUATION DE VLASOV
}

\author{
Martin Campos Pinto ${ }^{1}$
}

\begin{abstract}
In this talk, I present an adaptive semi-lagrangian scheme recently developed in collaboration with Michel Mehrenberger for approximating the solutions to the Vlasov-Poisson equation, and where the main feature consists in a new algorithm for transporting the multiscale meshes along the numerical flow. While reasonably simple, the algorithm we propose allows to transport "at first guess" the numerical solution to a given nonlinear transport problem, and the adaptive mesh on which this solution is computed. Moreover, this evolution is done in a way that is in some sense optimal, since on an analytical perspective, the accuracy of the solutions is established, together with a (still incomplete) bound on the complexity of the meshes. First proposed and analyzed in the article [3], then roughly described in a previous proceeding [5], this scheme was also given a detailed presentation in my $\mathrm{PhD}$ dissertation [4], in french. I shall here follow the latter and consider some "abstract", Vlasov-type problem which properties will first be recalled. I will then describe our algorithm for transporting the multiscale meshes, and explain how its main properties enter the error analysis of the numerical scheme.
\end{abstract}

Résumé. Dans cet exposé, je présente un schéma adaptatif semi-lagrangien développé récemment avec Michel Mehrenberger pour approcher les solutions de l'équation de Vlasov-Poisson, où l'ingrédient principal consiste en un nouvel algorithme qui transporte les maillages multi-échelles le long du flot numérique. Tout en étant relativement simple, notre algorithme permet en effet de transporter "du premier coup" la solution numérique du problème de transport non-linéaire et le maillage adaptatif sur lequel cette solution est calculée. Ceci en garantissant d'une part la précision des solutions, d'autre part l'optimalité des maillages ainsi construits, du moins dans une certaine mesure. D'abord proposé et analysé dans l'article [3], puis décrit de façon plus rapide dans la note [5], ce schéma a également fait l'objet d'une présentation détaillée dans ma thèse [4]. C'est essentiellement l'articulation de cette dernière que je suivrai ici, en considérant un problème de transport "abstrait" de type Vlasov dont j'énoncerai les principales propriétés. Je décrirai ensuite comment notre algorithme transporte les maillages adaptatifs multi-échelles, et j'indiquerai de quelle façon ses propriétés interviennent dans l'analyse d'erreur du schéma numérique global.

\section{INTRODUCTION}

Le principe de la démarche semi-lagrangienne, qu'il convient sans doute de rappeler, consiste à projeter à chaque pas de temps, et sur un maillage connu, la solution numérique du pas de temps précédent préalablement transportée le long des trajectoires caractéristiques du problème, ces trajectoires étant elles-mêmes approchées selon un schéma particulier. On pourra lire, pour une présentation plus détaillée, les articles [6] et [11]. Mais

\footnotetext{
${ }^{1}$ IGPM, RWTH, 56062 AACHEN, ALLEMAGNE
} 
d'une certaine façon, on peut déjà voir dans cette démarche l'alternance successive des discrétisations temporelle et spatiale respectivement matérialisées par l'approximation numérique des trajectoires d'une part, et la projection sur un espace de type éléments finis d'autre part. En termes plus schématiques, on écrira : $f^{n} \rightarrow \mathcal{T} f^{n} \rightarrow f^{n+1}:=P \mathcal{T} f^{n}$, un point crucial étant que la solution "intermédiaire" $\mathcal{T} f^{n}$ n'est en réalité connue qu'au travers des formes linéaires associées à la projection $P$ (de ses valeurs ponctuelles, pour une interpolation).

Dans notre schéma, comme dans d'autres qui l'ont précédé (voir notamment [2], [9] ou [10]), l'adaptativité consiste alors à faire évoluer "en même temps" que la solution numérique le maillage sur lequel cette solution est projetée, le but étant d'économiser des ressources de calcul en un maillage qui lui est bien adapté. La principale difficulté vient alors de la nécessité, pour projeter la solution transportée $\mathcal{T} f^{n}$, de connaître à l'avance le maillage qui lui est bien adapté : on parle alors de prédiction de maillage. Pour mettre en avant, dans cet exposé, la technique développée avec Michel Mehrenberger pour faire évoluer ces maillages adaptatifs, je considérerai un problème de transport non-linéaire "abstrait"

$$
\partial_{t} f(t, x, v)+F(t, x, v) \cdot \nabla_{x, v} f(t, x, v)=0, \quad t>0, \quad(x, v) \in \mathbb{R}^{2}
$$

où le terme de force $F$ est couplé avec $f$ (lire à ce sujet la remarque (1.1) ci-dessous) et dont les propriétés s'inspirent de celles de l'équation de Vlasov, lorsque la donnée initiale $f(0, \cdot)=f_{0}$ est suffisamment régulière . D'autre part, je supposerai connue une discrétisation en temps de ce problème, c'est-à-dire une façon d'approcher les trajectoires caractéristiques suivies par la solution exacte entre deux instants successifs $t_{n}:=n \Delta t$ et $t_{n+1}$.

Une fois fixée la structure générale des éléments finis, c'est bien entendu à partir de ces trajectoires approchées que sera construit le schéma adaptatif. Je commencerai donc par énoncer une série de propriétés (hypothèses de travail numérotées de (HT.1) à (HT.5)) vérifiées par ces trajectoires, avant de décrire l'algorithme qui fait évoluer les maillages d'un pas de temps à l'autre. J'introduirai alors un opérateur qui à un maillage adaptatif donné en associera un autre, en "suivant" le flot numérique formé par les trajectoires. Aussi, et bien que cet opérateur ne transporte pas les mailles de façon exacte (on verra pourquoi dans la suite), j'en parlerai comme d'un opérateur de transport de maillages. Enfin, et sous réserve de vérifier les hypothèses (HT.1)-(HT.5), j'établirai un résultat de précision uniforme a priori pour ce schéma adaptatif, ainsi qu'une analyse partielle de sa complexité.

\section{LE PROBLÈME ET SA DISCRÉTISATION}

\subsection{Approximation lagrangienne du transport non-linéaire}

Dans le problème (1), la non-linéarité vient du fait que le terme de force $F: \mathbb{R}_{+} \times \mathbb{R}^{2} \rightarrow \mathbb{R}^{2}$ est couplé avec $f$. Toutefois, et conformément à ce qui se passe pour l'équation de Vlasov, je considérerai que pour une donnée initiale lipschitzienne (ou même continue), la solution $f$ existe de façon unique sur tout intervalle de temps $[0, T]$, et plus précisément, qu'elle est transportée entre les instants $t_{n}=n \Delta t$ et $t_{n+1}$ le long de trajectoires caractéristiques

$$
t \rightarrow(X(t), V(t))=\left(X\left(t ; t_{n}, x, v\right), V\left(t ; t_{n}, x, v\right)\right)
$$

définies pour tout triplet $(n, x, v)$ comme solutions du système différentiel

$$
\partial_{t}(X(t), V(t))=F(t, X(t), V(t)), \quad\left(X\left(t_{n}\right), V\left(t_{n}\right)\right)=(x, v) .
$$

Remarque 1.1 (approximation explicite des trajectoires). Dans l'équation de Vlasov-Poisson, le terme de force s'écrit $F(t, x, v)=(v, E(t, x))$, où $E(t, x)$ désigne un champ électrique indépendant de la vitesse des particules. En l'absence de champ extérieur, le couplage entre " $F$ " et $f$ prend alors la forme $E(t, x)=\nabla_{x} \phi(t, x)$ et $\Delta_{x} \phi(t, x)=\rho(t, x)=\int f(t, x, v) \mathrm{d} v$, i.e. $E$ dérive d'un potentiel $\phi$ créé par la densité "physique" de charge. En particulier, l'expression (2)-(3) des trajectoires caractéristiques à partir de laquelle on fera évoluer $f$ entre 
les instants $t_{n}$ et $t_{n+1}$ fait intervenir $f$ de façon implicite dans ce même laps de temps. Dans la suite de cet exposé (comme dans [1], [3] et [4]), ces trajectoires seront approchées par un schéma explicite où n'interviennent que les valeurs de la solution au départ du pas de temps (et que je rappelerai, à titre d'information, dans la remarque 1.4 ci-dessous).

En ce qui concerne la régularité de $f$, j'admettrai qu'elle est également (et uniformément) lipschitzienne, au sens où

$$
\sup _{0<t<T}\|f(t)\|_{W^{1, \infty}\left(\mathbb{R}^{2}\right)} \leq C_{T}\left\|f_{0}\right\|_{W^{1, \infty}\left(\mathbb{R}^{2}\right)}
$$

avec une constante $C_{T}$ qui ne dépend que de l'instant final $T$. En désignant par $\mathcal{A}^{\text {exact }}\left[f\left(t_{n}\right)\right]$ le flot caractéristique entre $t_{n}$ et $t_{n+1}$, i.e.

$$
\mathcal{A}^{\text {exact }}\left[f\left(t_{n}\right)\right]=\mathcal{A}_{\Delta t}^{\text {exact }}\left[f\left(t_{n}\right)\right]:(x, v) \rightarrow\left(X\left(t_{n+1} ; t_{n}, x, v\right), V\left(t_{n+1} ; t_{n}, x, v\right)\right),
$$

l'opérateur de transport exact associé au problème (1) prend alors la forme

$$
\mathcal{T}^{\text {exact }}=\mathcal{T}_{\Delta t}^{\text {exact }}: f\left(t_{n}\right) \rightarrow f\left(t_{n+1}\right)=f\left(t_{n}\right) \circ\left(\mathcal{A}^{\text {exact }}\left[f\left(t_{n}\right)\right]\right)^{-1}
$$

Je supposerai enfin qu'on dispose d'une discrétisation en temps de ce problème i.e. d'un opérateur de transport approché

$$
\mathcal{T}=\mathcal{T}_{\Delta t}: f\left(t_{n}\right) \rightarrow f\left(t_{n}\right) \circ\left(\mathcal{A}\left[f\left(t_{n}\right)\right]\right)^{-1} \approx f\left(t_{n+1}\right)
$$

où $\mathcal{A}\left[f\left(t_{n}\right)\right]=\mathcal{A}_{\Delta t}\left[f\left(t_{n}\right)\right]$ est un difféomorphisme de $\mathbb{R}^{2}$ dans lui-même qui approche le flot exact $\mathcal{A}^{\text {exact }}\left[f\left(t_{n}\right)\right]$.

Remarque 1.2. L'opérateur $\mathcal{T}$ est ici présenté comme s'il agissait sur la solution exacte $f\left(t_{n}\right)$. De même, sa précision sera bientôt exprimée par l'hypothèse (HT.1) en utilisant cette solution exacte. Dans le schéma numérique, $\mathcal{T}$ ne pourra évidemment s'appliquer qu'à la solution approchée. Il conviendra donc de prendre cette différence en compte lors de l'analyse d'erreur, dans la section 3.

Remarque 1.3 (non-linéarité des opérateurs de transport). L'équation (1) étant non-linéaire, il en est de même pour les opérateurs de transport $\mathcal{T}^{\text {exact }}$ et $\mathcal{T}$. En ce qui concerne les flots caractéristiques (exacts ou approchés), ceci se manifeste par une dépendance vis-à-vis d'une "donnée de départ" lipschitzienne : ainsi, $\mathcal{A}[g]$ désigne le flot "issu" de la donnée $g$. Je noterai donc $\mathcal{A}[\cdot]$ l'opérateur de déplacement approché défini par (5), la lettre $\mathcal{A}$ seule désignant un difféomorphisme quelconque de $\mathbb{R}^{2}$ (éventuellement donné par $\mathcal{A}[g]$ ). Parallèlement, $\mathcal{T}[g]: \tilde{g} \rightarrow \tilde{g} \circ(\mathcal{A}[g])^{-1}$ désignera l'opérateur de transport linéaire associé à $g$, en observant que $\mathcal{T}$ vérifie $\mathcal{T} g=\mathcal{T}[g] g$.

Remarque 1.4 (approximation explicite des trajectoires, suite). Comme on l'a indiqué dans la remarque 1.1 cidessus, l'approximation des trajectoires repose sur une "simplification" de la dépendance entre le champ $F$ et la densité $f$. Ainsi, le schéma utilisé dans [1], [3] et [4] utilise une technique de splitting étudiée en 1976 par Cheng et Knorr (voir [6]). Les trajectoires y sont approchées en alternant les directions, par $\mathcal{A}_{x}=\mathcal{A}_{x, \Delta t / 2}:(x, v) \rightarrow$ $(x+v \Delta t / 2, v)$ et $\mathcal{A}_{v}[g]=\mathcal{A}_{v, \Delta t}[g]:(x, v) \rightarrow(x, v+\Delta t \tilde{E}[g](x))$ (où $\tilde{E}[g]$ désigne le champ électrique associé à une densité $g)$. L'opérateur $\mathcal{T}$ est alors obtenu en composant les transports uni-directionnels $\mathcal{T}_{x}: g \rightarrow g \circ\left(\mathcal{A}_{x}\right)^{-1}$ et $\mathcal{T}_{v} g=g \circ\left(\mathcal{A}_{v}[g]\right)^{-1}$, suivant $\mathcal{T}=\mathcal{T}_{x} \mathcal{T}_{v} \mathcal{T}_{x}$.

\subsection{Précision et régularité du transport approché}

Pour présenter notre schéma adaptatif semi-lagrangien, je vais maintenant énoncer plusieurs hypothèses "de travail", qui sont toutes vérifiées (lire [4]) lorsque l'équation de Vlasov-Poisson est approchée par un schéma temporel splitting. Ainsi, ma première hypothèse porte sur l'ordre de la discrétisation en temps. Plus précisément, je supposerai qu'il existe un $\sigma>0$ pour lequel

$$
\left\|f\left(t_{n+1}\right)-\mathcal{T} f\left(t_{n}\right)\right\|_{L^{\infty}} \leq c_{1} \Delta t^{\sigma+1} .
$$


En utilisant (4), on pourra remarquer qu'il suffit pour cela que les déplacements approchés vérifient (le terme "déplacement" étant ici pris dans un sens très large de "transformation du plan"), en notant $|x|:=\max _{i}\left|x_{i}\right|$,

$$
\sup _{x \in \mathbb{R}^{2}}\left|\left(\mathcal{A}\left[f\left(t_{n}\right)\right]\right)^{-1}(x)-\left(\mathcal{A}^{\text {exact }}\left[f\left(t_{n}\right)\right]\right)^{-1}(x)\right| \leq C \Delta t^{\sigma+1} .
$$

Je supposerai également que les déplacements $\mathcal{A}[g]$ et $(\mathcal{A}[g])^{-1}$ sont uniformément lipschitziens. Plus précisément, qu'il existe pour toute fonction $g \in W^{1, \infty}\left(\mathbb{R}^{2}\right)$ une constante $c_{2}$ telle que

$$
\left|\mathcal{A}[g](x)-\mathcal{A}[g]\left(x^{\prime}\right)\right| \leq c_{2}\left|x-x^{\prime}\right|
$$

et une constante $c_{3}$ telle que

$$
\left|(\mathcal{A}[g])^{-1}(x)-(\mathcal{A}[g])^{-1}\left(x^{\prime}\right)\right| \leq c_{3}\left|x-x^{\prime}\right| \quad \text { avec } \quad c_{3}<2 .
$$

Remarque 1.5. Dans notre schéma, cette hypothèse (HT.3) est vérifiée dès que le pas de temps $\Delta t$ est inférieur à une constante qui ne dépend que de la donnée initiale $f_{0}$ et du temps final $T=N \Delta t$. En particulier, les pas de temps ne sont pas soumis à une condition de type Courant-Friedrichs-Lewy, difficilement compatible avec la présence de mailles arbitrairement fines.

Enfin, le transport approché doit être " $\Delta t$-stable" vis-à-vis des perturbations de densité :

$$
\left\|\left[\mathcal{T}\left[g_{1}\right]-\mathcal{T}\left[g_{2}\right]\right] g_{3}\right\|_{L^{\infty}} \leq c_{4} \Delta t\left|g_{3}\right|_{W^{1, \infty}}\left\|g_{1}-g_{2}\right\|_{L^{\infty}} .
$$

Remarque 1.6. Les hypothèses (HT.1) et (HT.4) correspondent à une précision globale d'ordre $\sigma$ pour le schéma semi-discret $f_{\tau}^{0}:=f_{0}$, et pour $n=0, \ldots, N-1: f_{\tau}^{n+1}:=\mathcal{T} f_{\tau}^{n}$. On a en effet

$$
\left\|f(N \Delta t)-f_{\tau}^{N}\right\|_{L^{\infty}} \leq C \Delta t^{\sigma}
$$

après $N$ pas de temps, la constante $C$ ne dépendant que de $c_{1}, c_{4}$, du temps final $N \Delta t$ et de $f_{0}$. Pour le voir, on peut "fixer la linéarité" des opérateurs de transport en écrivant $\tilde{\mathcal{T}}^{n}=\mathcal{T}\left[f\left(t_{n}\right)\right]$ et $\mathcal{T}_{\tau}^{n}=\mathcal{T}\left[f_{\tau}^{n}\right]$, ce qui nous permet de décomposer $e_{n+1}^{\tau}:=\left\|f\left(t_{n+1}\right)-f_{\tau}^{n+1}\right\|_{L^{\infty}}$ suivant

$$
\begin{aligned}
e_{n+1}^{\tau} & \leq\left\|f\left(t_{n+1}\right)-\mathcal{T} f\left(t_{n}\right)\right\|_{L^{\infty}}+\left\|\left(\tilde{\mathcal{T}}^{n}-\mathcal{T}_{\tau}^{n}\right) f\left(t_{n}\right)\right\|_{L^{\infty}}+\left\|\mathcal{T}_{\tau}^{n}\left(f\left(t_{n}\right)-f_{\tau}^{n}\right)\right\|_{L^{\infty}} \\
& \leq c_{1} \Delta t^{\sigma+1}+\left(1+c_{4} \Delta t\left|f\left(t_{n}\right)\right|_{W^{1, \infty}}\right)\left\|f\left(t_{n}\right)-f_{\tau}^{n}\right\|_{L^{\infty}} \\
& \leq C \Delta t^{\sigma+1}+(1+C \Delta t) e_{n}^{\tau} .
\end{aligned}
$$

Pour la deuxième inégalité, on a utilisé les hypothèses (HT.1), (HT.4) et le fait qu'un transport n'augmente pas la norme $L^{\infty}$. Quant à la troisième, elle vient de l'hypothèse (4). L'estimation (6) découle alors du lemme de Gronwall.

\subsection{Maillages adaptatifs multi-échelles}

Dans notre schéma, la discrétisation spatiale met en œuvre des éléments finis de type $P_{1}$, au sens où chaque solution approchée est une fonction continue et affine par morceaux sur une triangulation sous-jacente. D'autre part, pour des raisons de simplicité algorithmique, nous avons choisi de n'utiliser que des triangulations basées sur des maillages quadrangulaires dyadiques et gradués.

J'en rappellerai brièvement la structure : 
- en premier lieu, les cellules de ces maillages sont des quadrangles dyadiques (isotropes) de la forme $\alpha_{\ell, k_{1}, k_{2}}:=\left[2^{-\ell} k_{1}, 2^{-\ell}\left(k_{1}+1\right)\left[\times\left[2^{-\ell} k_{2}, 2^{-\ell}\left(k_{2}+1\right)\left[\right.\right.\right.\right.$, où $\ell, k_{1}$ et $k_{2}$ sont des entiers qui représentent respectivement le niveau (ou l'échelle) et la position de la cellule,

- ensuite, ces maillages sont gradués au sens où deux cellules voisines (partageant une arête) doivent être de niveaux voisins.

Remarque 1.7. Les "maillages" dont il sera question dans la suite, et que je désignerai le plus souvent par la lettre $M$, seront tous de ce type.

Une propriété fondamentale de ces maillages, si l'on ne considère que des cellules de niveaux positifs, est qu'ils peuvent toujours être obtenus par une suite de raffinements locaux. Une façon élégante de le voir est de les munir d'une structure d'arbre, en désignant par $\mathcal{P}(\alpha)$ la parente d'une cellule $\alpha$, c'est-à-dire la cellule de niveau immédiatement inférieur contenant $\alpha$. Un ensemble $\Lambda$ de cellules dyadiques est alors un arbre lorsque le graphe formé par ces filiations est connexe, i.e. lorsque la parente de toute cellule de $\Lambda$ appartient également à $\Lambda$ (à l'exception, bien entendu des cellules de niveau 0). Clairement, un tel arbre n'est pas une partition, mais l'ensemble de ses feuilles "externes" $\partial \Lambda:=\{\alpha \notin \Lambda: \mathcal{P}(\alpha) \in \Lambda\}$ en est une, et il n'est pas difficile de voir que tout maillage (dyadique) $M$ est à son tour l'ensemble des feuilles externes de l'arbre $\Lambda(M):=\{\alpha: \exists \beta \in M, \beta \varsubsetneqq \alpha\}$. Dans ce cadre, le raffinement et le déraffinement de $M$ s'interprètent comme l'ajout ou le retrait d'une feuille (externe ou interne, respectivement) de $\Lambda(M)$, la propriété de graduation pouvant également s'exprimer comme une contrainte sur les arbres sous-jacents.

Je ne détaillerai pas ici la construction d'éléments finis de type $P_{1}$ à partir de ces maillages dyadiques gradués, renvoyant pour cela le lecteur à l'article [3] ou à ma thèse [4]. J'indiquerai simplement que cette construction est possible, et que la triangulation obtenue de cette façon est "équivalente" au maillage $M$ au sens où chaque triangle y intersecte un nombre fini de mailles, et réciproquement. L'interpolation affine par morceaux $P_{M}$ définie sur cette triangulation vérifie alors les propriétés suivantes :

(1) sa précision est contrôlée localement par la semi-norme $W^{2,1}$. Plus précisément, en désignant par $\mathcal{V}_{M}(\alpha):=\{\beta \in M: \bar{\alpha} \cap \bar{\beta} \neq \emptyset\}$ les cellules voisines de $\alpha$ dans le maillage $M$, on a

$$
\left\|\left(I-P_{M}\right) g\right\|_{L^{\infty}(\alpha)} \leq C|g|_{W^{2,1}\left(\mathcal{V}_{M}(\alpha)\right)},
$$

(2) elle est localement stable vis-à-vis de cette semi-norme, au sens où

$$
\left|P_{M} g\right|_{W^{2,1}(\alpha)} \leq C|g|_{W^{2,1}\left(\mathcal{V}_{M}(\alpha)\right)}
$$

En d'autres termes, la semi-norme $W^{2,1}$ constitue a priori un bon indicateur d'erreur pour décider de raffiner ou non un maillage dyadique lorsque l'erreur d'approximation est mesurée dans $L^{\infty}$. Pour les besoins de notre schéma itératif, il sera toutefois nécessaire d'utiliser un indicateur d'erreur applicable aux fonctions affines par morceaux (ce qui n'est pas le cas de la semi-norme $W^{2,1}$ ), et donc plus faible. Je noterai $\mathcal{E}(g, \alpha)$ ce nouvel indicateur, dont je ne détaillerai pas la forme pour ne pas alourdir cet exposé, renvoyant pour cela à l'article [3] (où il est noté $\nu$ ) ou à ma thèse [4], mais que je décrirai comme "proche" de la semi-norme $W^{2,1}$. En particulier, il est défini pour toute fonction $g$ (lipschitzienne) dont les dérivées secondes sont des mesures de Radon (ce qui est le cas des fonctions de $W^{2,1}$, ainsi que des fonctions affines par morceaux), et je désignerai dans la suite par $X \approx W^{1, \infty} \cap W^{2,1}$ l'espace de Banach contenant ces fonctions. Plus important, ce nouvel indicateur vérifie les propriétés suivantes :

(1) il contrôle les erreurs locales de projection, au sens où

$$
\left\|g-P_{M} g\right\|_{L^{\infty}(\alpha)} \leq C \sum_{\beta \in \mathcal{V}_{M}(\alpha)} \mathcal{E}(g, \beta) \quad \text { pour tout } \alpha \in M
$$


(2) pour toute fonction $g \in X, \mathcal{E}(g, \cdot)$ est sous-additif :

$$
\sum_{\beta, \mathcal{P}(\beta)=\alpha} \mathcal{E}(g, \beta) \leq \mathcal{E}(g, \alpha),
$$

(3) lorsque $\alpha$ est une cellule de $M, P_{M}$ est stable par rapport à $\mathcal{E}(\cdot, \alpha)$ :

$$
\mathcal{E}\left(P_{M} g, \alpha\right) \leq C \mathcal{E}(g, \alpha) .
$$

\subsection{Compatibilité du transport avec l'indicateur d'erreur}

La propriété que j'énonce à présent, fondamentale dans l'analyse du schéma, exprime la stabilité locale du transport approché vis-à-vis de l'indicateur d'erreur. Pour formuler cette stabilité, j'utiliserai la notion de domaine d'influence dans un maillage $M$ d'une cellule $\alpha$ vis-à-vis d'un flot $\mathcal{A}$ qui, rappelons-le, est un difféomorphisme de $\mathbb{R}^{2}$ dans lui-même. Une fois donnés ces différents objets, ce domaine est donc défini comme l'ensemble des cellules de $M$ dont l'image par le flot $\mathcal{A}$ intersecte $\alpha$, i.e.

$$
\mathcal{I}_{M, \mathcal{A}}(\alpha):=\{\beta \in M: \mathcal{A}(\beta) \cap \alpha \neq \emptyset\} \subset M .
$$

Remarque 1.8. Dans la mesure où $\alpha$ sera dans la suite une cellule potentielle d'un maillage prédit pour une solution à venir, il faudra voir $\mathcal{I}_{M, \mathcal{A}}$ comme un domaine d'influence dans le passé.

La propriété (minimale) de compatibilité portant sur le transport approché $\mathcal{T}: g \rightarrow g \circ(\mathcal{A}[g])^{-1}$ est alors qu'il existe une constante absolue $C$ telle que

$$
\mathcal{E}(\mathcal{T} g, \alpha) \leq C \sum_{\beta \in \mathcal{I}_{M, \mathcal{A}[g]}(\alpha)} \mathcal{E}(g, \beta)
$$

(on observera que le flot est bien "issu de $g$ "). Bien entendu, cette hypothèse n'a de sens que si l'indicateur d'erreur $\mathcal{E}$ est bien défini pour la fonction transportée $\mathcal{T} g$, ce qui sera le cas si $\mathcal{T}$ préserve la régularité $X$.

\section{Gestion dynamique des maillages multi-ÉCHelles}

A partir de l'estimation d'erreur (7), il est facile d'écrire un algorithme de découpage récursif $\boldsymbol{A}_{\varepsilon}$ qui à une fonction donnée $g$ associe le plus petit maillage dyadique sur lequel l'indicateur d'erreur $\mathcal{E}(g, \alpha)$ est uniformément majoré par un paramètre de tolérance $\varepsilon>0$ donné.

Algorithme $2.1\left(\boldsymbol{A}_{\varepsilon}: \varepsilon\right.$-adaptation à une fonction connue).

- Poser $\Lambda_{0}:=\left\{\alpha_{\left(0, k_{1}, k_{2}\right)}=\left[k_{1}, k_{1}+1\left[\times\left[k_{2}, k_{2}+1\left[:\left(k_{1}, k_{2}\right) \in \mathbb{Z}^{2}\right\}\right.\right.\right.\right.$.

- Pour $\ell \geq 0$, calculer

$$
\Lambda_{\ell+1}:=\Lambda_{\ell} \cup\left\{\alpha \in \partial\left(\Lambda_{\ell}\right): \mathcal{E}(g, \alpha)>\varepsilon\right\}
$$

jusqu'à ce que $\Lambda_{L+1}=\Lambda_{L}$, puis prendre $\tilde{M}=\partial \Lambda_{L}$.

- Définir $\boldsymbol{A}_{\varepsilon}(g)$ comme le plus petit raffinement gradué de $\tilde{M}$.

\subsection{Transport (prédiction) des maillages multi-échelles}

En suivant le même principe, on peut également écrire un algorithme de prédiction du maillage adaptatif, qui construira à partir d'une solution $g$ associée à un maillage $M$ un nouveau maillage, cette fois bien adapté à $\mathcal{T} g$. Comme on ne peut pas évaluer les quantités $\mathcal{E}(\mathcal{T} g, \alpha)$, l'idée consiste à s'appuyer sur l'adéquation du maillage $M$ avec $g$ et à transporter ce maillage le long du flot $\mathcal{A}[g]$ (sur lequel est défini $\mathcal{T} g$ ), ou plus précisément, pour 
obtenir un nouveau maillage dyadique, sa résolution.

Ainsi, pour un maillage donné $M$, j'appellerai niveau rétrograde d'une cellule $\alpha$ (relativement à un flot $\mathcal{A}$, et en notant $m_{\alpha} \in \mathbb{R}^{2}$ le centre de $\alpha$ ) l'entier

$$
\ell_{M, \mathcal{A}}^{*}(\alpha):=\max \left\{\ell(\beta): \beta \in M, \mathcal{A}^{-1}\left(m_{\alpha}\right) \in \bar{\beta}\right\}
$$

Observons que la plupart du temps, le point $\mathcal{A}^{-1}\left(m_{\alpha}\right)$ est à l'intérieur d'une cellule de $M$ et que le max est inutile. Avec ces notations, l'algorithme $\boldsymbol{T}[\mathcal{A}]$ de "transport" de maillages multi-échelles prend la forme suivante.

Algorithme 2.2 $(\boldsymbol{T}[\mathcal{A}]$ : transport de maillages dyadiques).

- Poser $\Lambda_{0}:=\left\{\alpha_{\left(0, k_{1}, k_{2}\right)}=\left[k_{1}, k_{1}+1\left[\times\left[k_{2}, k_{2}+1\left[:\left(k_{1}, k_{2}\right) \in \mathbb{Z}^{2}\right\}\right.\right.\right.\right.$.

- Pour $\ell \geq 0$, calculer

$$
\Lambda_{\ell+1}:=\Lambda_{\ell} \cup\left\{\alpha \in \partial\left(\Lambda_{\ell}\right): \ell_{M, \mathcal{A}}^{*}(\alpha)>\ell(\alpha)\right\}
$$

jusqu'à ce que $\Lambda_{L+1}=\Lambda_{L}$, et prendre $\tilde{M}=\partial \Lambda_{L}$.

- Définir $\boldsymbol{T}[\mathcal{A}] M$ comme le plus petit raffinement gradué de $\tilde{M}$.

En d'autres termes, le critère utilisé ici pour savoir si une maille doit ou non être subdivisée est que son niveau doit toujours être plus grand que celui de la cellule à laquelle appartient l'antécédent de son centre par le flot $\mathcal{A}$. Toutefois, pour que le maillage gradué $\boldsymbol{T}[\mathcal{A}] M$ construit de cette façon vérifie bien $\ell_{M, \mathcal{A}}^{*},(\alpha) \leq \ell(\alpha)$ sur chacune de ses mailles, il est nécessaire que cette propriété (clairement vraie pour $\tilde{M}$ ) soit préservée par le raffinement, autrement dit qu'elle vérifie une forme de monotonie. Le lemme suivant montre que c'est le cas, sous la condition de stabilité (HT.3).

Lemme 2.3. Soient $M$ un maillage dyadique gradué et $\mathcal{A}$ un flot vérifiant l'hypothèse (HT.3). Si l'on a $\ell_{M, \mathcal{A}}^{*}(\beta) \leq \ell(\beta)$ pour une cellule $\beta$, alors on a également

$$
\ell_{M, \mathcal{A}}^{*}(\alpha) \leq \ell(\alpha) \text { pour toute cellule } \alpha \subset \beta
$$

La preuve de ce lemme est donnée dans ma thèse, voir [4], lemme 5.7.

\subsection{Propriétés des maillages transportés}

Les propriétés essentielles de notre algorithme $\boldsymbol{T}[\mathcal{A}]$ sont exprimées par :

- le théorème 2.4, qui exprime le fait que l'ordre de complexité des maillages est préservé par l'algorithme,

- la proposition 2.5, qui garantit que les domaines d'influence (9) associés aux cellules de $\boldsymbol{T}[\mathcal{A}] M$ ont un cardinal uniformément borné. Associé à la stabilité (HT.5) du transport vis-à-vis de $\mathcal{E}$, ce résultat permettra d'établir dans la section 3.2 une estimation d'erreur.

Théorème 2.4 (complexité des maillages transportés). Si $\mathcal{A}$ et $\mathcal{A}^{-1}$ sont tous les deux lipschitziens, alors il existe une constante $C$ pour laquelle

pour tout maillage $M$ (dyadique et gradué).

$$
\#(\boldsymbol{T}[\mathcal{A}] M) \leq C \#(M)
$$

La preuve de ce théorème est donnée dans ma thèse, voir [4], théorème 5.1.

Proposition 2.5 (stabilité du transport de maillages dyadiques). Si $\mathcal{A}$ vérifie l'hypothèse (HT.3) alors il existe deux constantes $c_{6}$ et $c_{7}$ dépendant uniquement de $c_{3}$ pour lesquelles on a (pour tout maillage $M$ )

$$
\sup _{\beta \in \mathcal{I}_{M, \mathcal{A}}(\alpha)} \ell(\beta) \leq \ell(\alpha)+c_{6}, \quad \alpha \in \boldsymbol{T}[\mathcal{A}] M,
$$




$$
\#\left(\mathcal{I}_{M, \mathcal{A}}(\alpha)\right) \leq c_{7}, \quad \alpha \in \boldsymbol{T}[\mathcal{A}] M
$$

Preuve. Soit $M$ un maillage dyadique et $\alpha$ une cellule fixée de $\boldsymbol{T}[\mathcal{A}] M$. Si $\eta \in M \operatorname{contient} \mathcal{A}^{-1}\left(m_{\alpha}\right)$, on a par construction

$$
\ell(\eta) \leq \ell(\alpha) .
$$

Si d'autre part $\beta \in \mathcal{I}_{M, \mathcal{A}}(\alpha)$, il existe un point $m \in \alpha$ tel que $\mathcal{A}^{-1}(m) \in \beta$. L'hypothèse (HT.3) entraîne alors

$$
\left|\mathcal{A}^{-1}\left(m_{\alpha}\right)-\mathcal{A}^{-1}(m)\right| \leq c_{3}\left|m_{\alpha}-m\right| \leq c_{3} 2^{-\ell(\alpha)-1},
$$

ce qui signifie que $\beta$ intersecte la boule $B_{\alpha}^{*}$ dont le centre $\mathcal{A}^{-1}\left(m_{\alpha}\right)$ appartient à $\eta$, et dont le rayon vaut $r_{\alpha}^{*}=c_{3} 2^{-\ell(\alpha)-1} \leq c_{3} 2^{-\ell(\eta)-1}$ (il s'agit d'une boule "carrée", pour la distance $\ell^{\infty}$ de $\mathbb{R}^{2}$ ). Comme $\beta$ et $\eta$ font toutes deux parties du même maillage gradué $M$, on peut voir sur la figure 1 que l'hypothèse $c_{3} 2^{-\ell(\eta)-1}<2^{-\ell(\eta)}$ va entraîner d'une part, que le niveau de $\beta$ ne peut pas être arbitrairement supérieur à celui de $\eta$, et d'autre part que le nombre de cellules $\beta$ est effectivement borné de façon indépendante de $\alpha$. Plus précisément, on peut montrer (en utilisant le fait que $M$ est gradué) que si $\delta:=\ell(\beta)-\ell(\eta) \geq 1$, alors

$$
2^{-\ell(\eta)}\left[1-2^{1-\delta}\right] \leq\left|\mathcal{A}^{-1}\left(m_{\alpha}\right)-\mathcal{A}^{-1}(m)\right| .
$$

On en déduit en utilisant (13) que $1-2^{1-\delta} \leq c_{3} / 2$, ce qui implique $\delta$ est inférieur à $2-\ln _{2}\left(2-c_{3}\right)$. En utilisant (12), ceci implique déjà (10) avec $c_{6}=2-\ln _{2}\left(2-c_{3}\right)$. Revenant au nombre maximal de cellules $\beta$, on peut alors vérifier qu'il est inférieur à $c_{7}=9 \times 2^{2 \delta}$ (en considérant (i) que ce nombre est maximal dans le cas où la graduation de $M$ est "saturée" autour de $\eta$, (ii) qu'en raison de $r_{\alpha}^{*}<2^{-\ell(\eta)}, B_{\alpha}^{*}$ intersecte au plus 9 "super-cellules" de niveau $\ell(\eta)$, et (iii) que chacune de ces "super-cellules" contient au plus $2^{2 \delta}$ cellules de niveau $\ell(\eta)+\delta)$.

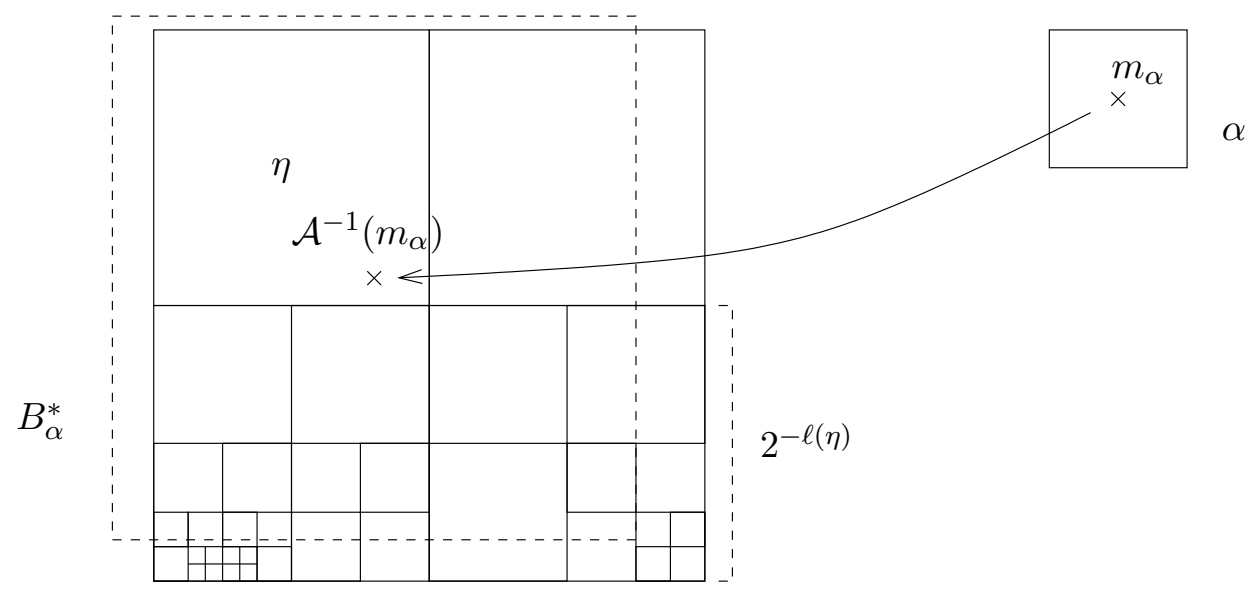

Figure 1. les cellules de $\mathcal{I}_{M, \mathcal{A}}(\alpha)$ intersectent toutes la "boule" $B_{\alpha}^{*}$.

\subsection{Le schéma complet de transport par prédiction et correction}

Je désigne à présent par $\left(f^{n}, M^{n}\right)$ la solution numérique adaptative (et son maillage) au pas de temps $n$. Le schéma est alors déterminé par deux paramètres scalaires : le pas de temps constant $\Delta t$, et une tolérance $\varepsilon>0$ qui représente à chaque pas de temps l'erreur $L^{\infty}$ autorisée pour les approximations adaptatives. 
La solution numérique initiale est obtenue en projetant la donnée initiale exacte $f_{0}$ sur le plus petit maillage dyadique qui lui est $\varepsilon$-adapté au sens de l'indicateur d'erreur $\mathcal{E}$. En utilisant l'algorithme $\boldsymbol{A}_{\varepsilon}$, j'ai ainsi

$$
M^{0}:=\boldsymbol{A}_{\varepsilon}\left(f_{0}\right), \quad f^{0}:=P_{M^{0}} f_{0}
$$

Au pas de temps $n$, et en désignant par $\mathcal{A}^{n}:=\mathcal{A}\left[f^{n}\right]$ le flot associé au transport approché (5), la solution $\left(M^{n+1}, f^{n+1}\right)$ est alors calculée en deux étapes. Dans un premier temps, un maillage "intermédiaire" $\tilde{M}^{n+1}$ est prédit en transportant $M^{n}$ via $\boldsymbol{T}\left[\mathcal{A}^{n}\right]$, et c'est sur ce maillage que la solution numérique $f^{n}$ est transportée. Dans un deuxième temps, ce maillage intermédiaire est corrigé à partir de la solution obtenue, notamment pour garantir une précision constante des approximations au cours du temps (on reviendra sur ce point dans la section suivante). En d'autres termes, notre schéma calcule :

$$
\begin{aligned}
\tilde{M}^{n+1}:=\boldsymbol{T}\left[\mathcal{A}^{n}\right] M^{n}, & \tilde{f}^{n+1}:=P_{\tilde{M}^{n+1}} \mathcal{T} f^{n}, \\
M^{n+1}:=\boldsymbol{A}_{\varepsilon}\left(\tilde{f}^{n+1}\right), & f^{n+1}:=P_{M^{n+1}} \tilde{f}^{n+1} .
\end{aligned}
$$

En particulier, on observera qu'il n'est jamais nécessaire de réappliquer l'opérateur de transport $\mathcal{T}$ sur un maillage corrigé, la raison étant que notre algorithme de prédiction $\boldsymbol{T}\left[\mathcal{A}^{n}\right]$, comme on va bientôt le voir, génère des maillages suffisamment précis.

\section{Analyse D'erreur}

\subsection{Analyse intuitive}

A chaque pas de temps, la solution numérique est donc obtenue par un schéma de "transport-projection"

$$
f^{n+1}=P_{M^{n+1}} P_{\tilde{M}^{n+1}} \mathcal{T} f^{n},
$$

tandis que les maillages dyadiques sur lesquels repose la discrétisation adaptative sont obtenus par un schéma de "transport-correction". On peut en donner une vision synthétique en désignant par

$$
\mathcal{E}_{\infty}(g, M):=\sup _{\alpha \in M} \mathcal{E}(g, \alpha)
$$

l'adéquation globale entre $g$ et $M$, quantité qui vérifie notamment

$$
\left\|g-P_{M} g\right\|_{L^{\infty}} \leq C \mathcal{E}_{\infty}(g, M)
$$

d'après la propriété (7) et le fait que les voisines d'une cellule donnée sont toujours en nombre borné (par une constante absolue) dans un maillage gradué. Par construction, le maillage $\boldsymbol{A}_{\varepsilon}(g)$ créé par l'algorithme 2.1 vérifie alors

$$
\mathcal{E}_{\infty}\left(g, \boldsymbol{A}_{\varepsilon}(g)\right) \leq \varepsilon,
$$

et sous réserve que le transport approché (5) vérifie certaines des hypothèses (HT.i) énoncées plus haut, on peut montrer que $\boldsymbol{T}\left[\mathcal{A}^{n}\right]$ préserve l'ordre d'adéquation au cours du transport, i.e. que l'on a

$$
\mathcal{E}_{\infty}\left(\mathcal{T} f^{n}, \boldsymbol{T}\left[\mathcal{A}^{n}\right] M^{n}\right) \leq C \mathcal{E}_{\infty}\left(f^{n}, M^{n}\right)
$$

pour une constante $C$ indépendante du pas de temps $n$. En revanche, rien n'empêche a priori cette constante $C$ d'être supérieure à 1 , et donc l'adéquation globale maillage-solution de se détériorer fortement au cours de la simulation. On verra donc le pas (15a) comme une méthode simple permettant de faire évoluer le maillage entre deux instants $t_{n}$ et $t_{n+1}$, avec une précision acceptable sur quelques itérations (et qui préserve, d'après le théorème 2.4, l'ordre de complexité des maillages), mais insuffisante à plus long terme. En particulier, la 
résolution maximale n'augmente pas lorsqu'on passe de $M^{n}$ à $\boldsymbol{T}\left[\mathcal{A}^{n}\right] M^{n}$, alors que la régularité des solutions peut se dégrader fortement au cours de la simulation. Le pas (15b) permet alors de ramener l'adéquation du maillage en-dessous du paramètre de tolérance $\varepsilon$, et ceci de façon optimale, dans le cadre offert par les maillages dyadiques.

En ce qui concerne la complexité de notre schéma, son analyse (encore inachevée) tient essentiellement en deux arguments : le premier est exprimé par le théorème 2.4, selon lequelle notre algorithme de transport dyadique $\mathbf{T}\left[\mathcal{A}^{n}\right]$ préserve l'ordre de complexité des maillages comme on vient de le rappeler, mais ne l'empêche pas d'augmenter. A nouveau, on dispose donc d'une stabilité acceptable sur quelques itérations, mais insuffisante à long terme. En particulier, la taille des maillages transportés peut augmenter de façon exponentielle avec le nombre d'itérations, sans aucun rapport avec le pas de temps ou avec les propriétés d'approximation de la solution exacte $f$. Le deuxième argument consiste alors à estimer la complexité du maillage $\boldsymbol{A}_{\varepsilon}(g)$ à partir de la régularité de $g$, et à estimer cette régularité au cours du schéma numérique.

\subsection{Résultat principal}

Théorème 3.1 (Précision des solutions). Si le transport approché (5) satisfait les hypothèses (HT.1)-(HT.5) l'erreur numérique $e_{n}:=\left\|f\left(t_{n}\right)-f^{n}\right\|_{L^{\infty}}$ associée au schéma (14)-(15) vérifie

$$
e_{N} \leq C\left(\Delta t^{\sigma}+\varepsilon \Delta t^{-1}+\varepsilon\right)
$$

la constante $C$ ne dépendant que des constantes $c_{1}, \ldots, c_{4}$ apparaissant dans (HT.1)-(HT.4), du temps final $T=N \Delta t$ et de la solution initiale $f_{0}$.

Proof. D'après les estimations (18) et (19), l'erreur initiale $e_{0}:=\left\|\left(I-P_{M^{0}}\right) f_{0}\right\|_{L^{\infty}}$ se majore sans peine par $C \varepsilon$. La forme (16) du schéma nous permet alors de décomposer l'erreur $e_{n+1}$ en

$$
e_{n+1} \leq\left\|f\left(t_{n+1}\right)-\mathcal{T} f\left(t_{n}\right)\right\|_{L^{\infty}}+\left\|\mathcal{T} f\left(t_{n}\right)-\mathcal{T} f^{n}\right\|_{L^{\infty}}+\left\|(\tilde{P}-I) \mathcal{T} f^{n}\right\|_{L^{\infty}}+\left\|(P-I) \tilde{f}^{n+1}\right\|_{L^{\infty}},
$$

où $P$ et $\tilde{P}$ désignent respectivement $P_{M^{n+1}}$ et $P_{\tilde{M}^{n+1}}$. Le premier terme est en quelque sorte "résolu", puisqu'il s'agit de l'erreur en temps majorée par l'hypothèse (HT.1). Le deuxième terme se majore comme dans la remarque 1.6, en exploitant conjointement la régularité lipschitzienne (4) de la solution exacte et la stabilité (HT.4) du transport $\mathcal{T}$ relativement aux perturbations de densité. On a ainsi, en posant $\tilde{\mathcal{T}}^{n}:=\mathcal{T}\left[f\left(t_{n}\right)\right]$ et $\mathcal{T}^{n}:=\mathcal{T}\left[f^{n}\right]$ - tous deux linéaires -

$$
\left\|\mathcal{T} f\left(t_{n}\right)-\mathcal{T} f^{n}\right\|_{L^{\infty}} \leq\left\|\mathcal{T}_{n}\left(f\left(t_{n}\right)-f^{n}\right)\right\|_{L^{\infty}}+\left\|\left(\tilde{\mathcal{T}}^{n}-\mathcal{T}^{n}\right) f\left(t_{n}\right)\right\|_{L^{\infty}} \leq(1+C \Delta t) e_{n}
$$

en utilisant (HT.4), (4), et le fait qu'un transport n'augmente pas la norme $L^{\infty}$.

Restent donc les erreurs de projection $\left\|(P-I) \tilde{f}^{n+1}\right\|_{L^{\infty}}$ et $\left\|(\tilde{P}-I) \mathcal{T} f^{n}\right\|_{L^{\infty}}$. D'après (17)-(18), elles seront contrôlées par $\varepsilon$ si l'on est capable de garantir respectivement

A1. que $\mathcal{E}\left(\tilde{f}^{n+1}, \alpha\right)$ est au plus de l'ordre de $\varepsilon$ pour toute cellule $\alpha \in M^{n+1}$,

A2. que $\mathcal{E}\left(\mathcal{T} f^{n}, \alpha\right)$ est au plus de l'ordre de $\varepsilon$ pour toute cellule $\alpha \in \tilde{M}^{n+1}$.

A nouveau, on insistera sur la différence entre les assertions A1 et A2. La première correspond à un problème d'adaptation statique, dont la solution naturelle consiste à prendre $M^{n+1}=\boldsymbol{A}_{\varepsilon}\left(\tilde{f}^{n+1}\right)$. La seconde, quant à elle, correspond à un problème dynamique pour lequel la solution précédente ne s'applique plus, dans la mesure où ne souhaitant pas calculer les valeurs de la solution transportée $\mathcal{T} f^{n}$ sur un maillage uniformément fin, on ne dispose pas en pratique de ses valeurs à ce stade du calcul. On peut alors montrer que la construction de $\tilde{M}^{n+1}$ par l'algorithme de prédiction $\boldsymbol{T}\left[\mathcal{A}^{n}\right]$ fournit une réponse satisfaisante, et la démonstration se fait en trois étapes : 
1. Adéquation du maillage de départ. En utilisant (8), (14) et (19), on voit que $\left(M^{0}, f^{0}\right)$ vérifie

$$
\mathcal{E}_{\infty}\left(f^{0}, M^{0}\right) \leq C \mathcal{E}_{\infty}\left(f_{0}, M^{0}\right) \leq C \varepsilon
$$

et pour $n \geq 1$, on obtient de la même façon grâce à (15b) que

$$
\mathcal{E}_{\infty}\left(f^{n}, M^{n}\right) \leq C \mathcal{E}_{\infty}\left(\tilde{f}^{n}, M^{n}\right) \leq C \varepsilon
$$

On en déduit que les maillages $M_{n}$ sont toujours bien adaptés aux solutions numériques $f_{n}$, au sens où l'on a

$$
\mathcal{E}_{\infty}\left(f^{n}, M^{n}\right) \leq C \varepsilon
$$

avec une constante absolue.

2. Evolution des indicateurs d'erreurs. Comme $\mathcal{T} f^{n}=f^{n} \circ\left(\mathcal{A}^{n}\right)^{-1}$, l'hypothèse de compatiblité (HT.5) nous permet d'écrire

$$
\mathcal{E}\left(\mathcal{T} f^{n}, \alpha\right) \leq C \sum_{\beta \in \mathcal{I}_{M^{n}, \mathcal{A}^{n}}(\alpha)} \mathcal{E}\left(f^{n}, \beta\right)
$$

pour toute cellule dyadique $\alpha$ (on ne fait ici que recopier une hypothèse, mais c'est en réalité une étape importante de l'analyse du schéma).

3. Contrôle des domaines d'influence. La dernière étape consiste à exploiter la propriéte (11) des maillages transportés. En vertu de l'hypothèse (HT.3), en effet, la proposition 2.5 s'applique et toute cellule $\alpha$ appartenant à $\tilde{M}^{n+1}=\boldsymbol{T}\left[\mathcal{A}^{n}\right] M^{n}$ vérifie

$$
\mathcal{E}\left(\mathcal{T} f^{n}, \alpha\right) \leq C \#\left(\mathcal{I}_{M^{n}, \mathcal{A}^{n}}(\alpha)\right) \mathcal{E}_{\infty}\left(f^{n}, M^{n}\right) \leq C \varepsilon
$$

pour tout entier $n$, en utilisant $(23)$, (11) et (22). On en déduit donc bien

$$
\mathcal{E}_{\infty}\left(\mathcal{T} f^{n}, \tilde{M}^{n+1}\right) \leq C \varepsilon
$$

ce qui répond au problème A2.

$\mathrm{Au}$ cours de cette analyse d'erreur, on a successivement vérifié que (i) l'erreur de discrétisation en temps $\left\|f\left(t_{n+1}\right)-\mathcal{T} f\left(t^{n}\right)\right\|_{L^{\infty}}$ était de l'ordre de $\Delta t^{\sigma+1}$, (ii) l'erreur de couplage $\left\|\mathcal{T} f\left(t_{n}\right)-\mathcal{T} f^{n}\right\|_{L^{\infty}}$ était inférieure à $(1+C \Delta t) e_{n}$, (iii) l'erreur de projection "statique" $\left\|\left(P_{M^{n+1}}-I\right) \tilde{f}^{n+1}\right\|_{L^{\infty}}$ était de l'ordre de $\varepsilon$, (iv) ainsi que l'erreur de projection "dynamique" $\left\|\left(P_{\tilde{M}^{n+1}}-I\right) \mathcal{T} f^{n}\right\|_{L^{\infty}}$. Compte tenu de la décomposition (21), on en déduit

$$
e_{n+1} \leq C\left(\Delta t^{\sigma+1}+\varepsilon\right)+(1+C \Delta t) e_{n}
$$

et finalement l'estimation (20) en utilisant un lemme de Gronwall discret.

\section{REFERENCES}

[1] N. BESSE "Convergence of a semi-Lagrangian scheme for the one-dimensional Vlasov-Poisson system" SIAM J. Numer. Anal., Vol. 42, No. 1, pp. 350-382 (2004).

[2] N. Besse, F. Filbet, M. Gutnic, I. Paun et E. Sonnendrücker "An adaptive numerical method for the Vlasov equation based on a multiresolution analysis" in Numerical Mathematics and Advanced Applications ENUMATH 2001 (F. Brezzi, A. Buffa, S. Escorsaro and A. Murli Ed.), Springer, pp. 437-446 (2001).

[3] M. Campos Pinto et M. Mehrenberger "Convergence of an adaptive scheme for the one-dimensional Vlasov-Poisson system", soumis à SIAM J. Numer. Anal. 
[4] M. Campos Pinto "Développement et analyse de schémas adaptatifs pour les équations de transport", Thèse de l'Université Pierre et Marie Curie, Paris, 2005.

[5] M. Campos Pinto "A direct and accurate adaptive semi-Lagrangian scheme for the Vlasov-Poisson equation", soumis à International Journal of Applied Mathematics and Computer Science.

[6] C.Z. Cheng et G. Knorr "The integration of the Vlasov equation in configuration space", J. Comput. Phys., Vol. 22, pp. 330-351 (1976).

[7] A. Cohen, S.M. Kaber, S. Müller et M. Postel "Fully adaptive multiresolution finite volume schemes for conservation laws" Math. Comp., Vol. 72, No. 241, pp. 183-225 (2003).

[8] R. DeVore "Nonlinear approximation" in Acta Numerica, Vol. 7, Cambridge Univ. Press, pp. 51-150 (1998).

[9] M. Gutnic, M. Haefele, I. Paun et E. Sonnendrücker "Vlasov simulations on an adaptive phase-space grid", Comput. Phys. Comm., Vol. 164, pp. 214-219 (2004).

[10] E. Sonnendrücker, F. Filbet, A. Friedman, E. Oudet et J.L. Vay "Vlasov simulation of beams with a moving grid" Comput. Phys. Comm., Vol. 164, pp. 390-395 (2004).

[11] E. Sonnendrücker, J. Roche, P. Bertrand et A. Ghizzo "The semi-Lagrangian method for the numerical resolution of the Vlasov equation" J. Comput. Phys., Vol. 149, No. 2, pp 201-220 (1999).

[12] H. Yserentant: "Hierarchical bases" in ICIAM 91 (Washington, DC, 1991), SIAM, Philadelphia, PA, pp. $256-276$ (1992). 